\title{
Effects of Exergaming on Balance of Healthy Older Adults: A Systematic Review and Meta-analysis of Randomized Controlled Trials
}

\author{
Qun Fang, MS, ${ }^{1}$ Parisa Ghanouni, PhD, ${ }^{2}$ Sarah E. Anderson, OTD, ${ }^{3}$ Hilary Touchett, $\mathrm{PhD},{ }^{4}$ \\ Rebekah Shirley, BS, ${ }^{1}$ Fang Fang, $\mathrm{MB}{ }^{5}$ and Chao Fang, $\mathrm{PhD}^{6}$
}

\begin{abstract}
Balance is critical for older adults to perform daily activities. However, age-related declines in balance increase the risk of falls and severe injuries, such as bone fractures and head injuries. Exergames have been widely applied to improve health-related outcomes in older adults. This meta-analysis aims to quantify the effects of exergaming interventions on balance performance in healthy older adults. A literature search was performed using PubMed, ScienceDirect, SPORTDiscus, COCHRANE, EBSCO, and EMBASE. A total of 16 experimental studies met inclusion criteria for a full-text review. Data synthesis examined balance functions, including static, dynamic, proactive, and perceived balance abilities when performing daily activities. Intervention protocols of the reviewed studies included an average of two to three 40-minute exergaming sessions per week for 8 weeks. A random effects model identified significant effects in favor of the exergaming group, with moderate effect size in dynamic balance (Hedges' $\mathrm{g}=0.36,95 \% \mathrm{CI}=0.26-1.30, P<0.001$ ), and perceived balance (Hedges' $\mathrm{g}=0.31,95 \% \mathrm{CI}=0.04-0.58, P=0.02$ ); and considerable effect size in Chair Stand Test (Hedges' $\mathrm{g}=0.78,95 \% \mathrm{CI}=0.26-1.30, P=0.003$ ), and balance test batteries (Hedges' $\mathrm{g}=0.72,95 \%$ $\mathrm{CI}=0.42-1.02, P<0.001$ ). No significant effect was found in the static balance (Hedges' $\mathrm{g}=0.22,95 \%$ $\mathrm{CI}=-0.31$ to $0.76, P=0.42$ ), or proactive balance (Hedges' $\mathrm{g}=0.54,95 \% \mathrm{CI}=-0.12$ to $1.20, P=0.11$ ). Metaanalysis identified exergaming-associated benefits in older adults' balance function and confidence. This finding supports the feasibility of exergaming as a supplementary approach to improve balance for healthy older adults. Health professionals may optimize treatment effect by integrating exergaming sessions into a traditional balance exercise program.
\end{abstract}

Keywords: Exergames, Videogames, Virtual reality, Balance, Older adults, Meta-analysis

\section{Introduction}

B ALANCE PERFORMANCE OFTEN decreases with aging. Skeletal muscular changes, such as reduction in muscle strength, muscle mass, power, and joint mobility, may contribute to the poor balance control in older adults. ${ }^{1}$ Additionally, age-related declines in the Central Nervous System exert a negative impact on an individual's capacity of maintaining balance in response to body perturbations. ${ }^{2}$ For example, rapid stepping or reaching movements are important strategies for fall prevention. ${ }^{1}$ However, deteri- oration in joint proprioception and neural connectivity inhibit accurate and prompt postural adjustment, increasing the risk of falls. 3,4

Falls are a major cause of bone fractures and head injuries in older adults, creating a public health concern. ${ }^{5}$ According to Centers for Disease Control and Prevention, the fall death rate for older adults in the United States has been increasing over the past decade from $47 \%$ in 2007 to $62 \%$ in $2016^{6}$ Falls can negatively impact psychological status even if the fall does not result in an injury. Older adults with fall experience tend to self-limit daily life-related physical activity

\footnotetext{
${ }^{1}$ Department of Kinesiology, Mississippi State University, Mississippi State, Mississippi.

${ }^{2}$ School of Occupational Therapy, Dalhousie University, Halifax, Nova Scotia.

${ }^{3}$ School of Health and Rehabilitation Sciences, The Ohio State University, Columbus, Ohio.

${ }^{4}$ Cizik School of Nursing, University of Texas Health Science Center at Houston, Houston, Texas.

${ }^{5}$ Rehabilitation Center, Qing Dao Fu Wai Hospital, Qingdao, China.

${ }^{6}$ Department of Pharmacology, the Fourth Military Medical University, Xi'an, China.
} 
due to fear of falling. Sedentary lifestyles worsen physical functions and further increase the chance of falling, leading to a vicious cycle. ${ }^{7,8}$ Moreover, the lack of confidence in balance control when performing daily activities results in greater dependence on others, in turn decreases quality of life in older adults with fall experience. 9,10

Regular exercises have proven effective in strengthening lower limb muscles, improving flexibility, and counteracting age-related decline in neuromotor and somatosensory functions. ${ }^{11-13}$ The benefits are critical in improving balance control and reducing fall risk. ${ }^{14,15}$ Standard balance exercise protocols based on functional activities and progressive resistance training have been applied to clinical rehabilitation. ${ }^{16-18}$ However, adherence to exercise programs is challenging, because exercise programs are often considered monotonous and boring. ${ }^{19,20}$ Loss of interest is a common reason for discontinuation of regular exercise programs among clients. ${ }^{20,21}$ Researcher demonstrates that benefits gained through previous training regress rapidly toward pretraining levels if older adults quit in the middle of an exercise program. $^{22}$ Therefore, there is a need to determine effective approaches to improving adherence to a taskoriented training and motivate self-regulated practice outside therapy sessions. $^{23}$

Exergames are interactive gaming systems which require participants' body movement during game play. ${ }^{24}$ Common gaming systems include Dance Dance Revolution, Xbox Kinect, and Nintendo Wii. Exergames have been widely applied as a means of providing moderate physical activities for people with special needs. ${ }^{25-27}$ Previous research suggests that exergames are safe and feasible for older adults. ${ }^{28}$ Exergaming allows participants to engage in physical activities in an enjoyable way. ${ }^{29}$ Older adults report positive experiences in game play as well as willingness to continue the activity after an exergaming intervention program. ${ }^{21,30,31}$

Given the motivations to engage in physical activities, researchers perceive exergaming as a potential solution to the adherence issue in practice. As exergames become increasingly popular, there is an increasing number of studies investigating effects of exergaming on balance control in older adults. In a literature review examining effects of Nintendo Wii games on older adults, researchers identified positive effects of improved balance, decreased depression, improved cognitive function, and enhanced quality of life in older adults. $^{20}$

Laufer and colleagues ${ }^{32}$ conducted a systematic review of exergaming-related effects on balance control in older adults. The study identified effects in favor of exergaming interventions compared with inactive control groups. However, the researchers pointed out that definitive recommendations could not be made at that time because of insufficient data as well as great variabilities in intervention protocols and outcome measures. Given the fact that a number of randomized controlled trials (RCTs) have been available since the systematic review was published in 2014, it is now feasible to summarize quantified evidence based on current experimental designs. By focusing on subjects' performance in a set of balance tests, which assess both functional performance and confidence in fall prevention, the current systematic review aims to investigate whether older adults significantly improve balance control after participating in exergaming interventions.

\section{Methods}

\section{Search strategy}

For this review, we conducted a literature search through major databases, including PubMed, SPORTDiscus, ScienceDirect, COCHRANE, EMBASE, and EBSCO from January 2000 to January 2019. To identify those studies that fit into the main purpose of the meta-analysis, the search focused on four constructs: exergaming, healthy older adults, balance, and experimental design. The following combinations of terms were used for literature search: "exergame* OR active video gam* OR virtual reality gam* OR serious gam* OR motion gam*" AND "older adult* OR elderly", AND "balance OR postural control" AND "randomized controlled trial OR RCT." To ensure an exhaustive search, colleagues of the authors as well as experts in exergaming intervention or balance control were consulted.

\section{Eligibility criteria}

Studies that met the following criteria were considered for inclusion: (1) peer-reviewed articles published in English; (2) exergaming platform applied to the intervention; (3) randomized design which involves comparisons between treatment and control group; and (4) outcome measures that include at least one balance assessment. Subjects of the included studies were healthy older adults (above 60 years of age) who could perform daily activities with no or minimal assistance. Healthy older adults were referred to having normal vision, no or minimal neurological deficits or cognitive impairments (Mini-Mental State Examination $>24$ ), being able to stand and walk without aid, and no severe cardiovascular or respiratory ailments.

Compared with other types of study design such as pre/ post study and quasiexperimental design, RCTs provide the most powerful evidence on the effects of a treatment intervention. ${ }^{33,34}$ Randomization reduces risk of bias in selection and allocation. In addition, similar conditions between control and treatment groups at the baseline enable researchers to attribute any between-group differences in outcome to the intervention. ${ }^{35}$ As a widely accepted "gold standard" for medical research, RCT is the only study design considered in the current systematic review. Accordingly, studies with the following features were excluded: (1) editorial or conference abstracts; (2) book chapters; (3) review articles; and (4) nonrandomized studies, including clinical controlled trials.

\section{Study selection}

A three-step screening process was performed to select studies meeting eligibility criteria. The initial screening removed irrelevant articles based on title examination. Next, researchers determined eligibility by reviewing abstracts of the articles. In the third step, a full-text evaluation was conducted to assess eligibility of the remaining articles. Two authors (H.T. and R.S.) worked independently to identify relevant articles. Any disagreement on the eligibility of an article was resolved by having a discussion with other authors during consensus meetings.

\section{Quality assessment and risk of bias}

Methodological quality of the eligible studies was assessed by the Physiotherapy Evidence Database (PEDro) 
scale, which is a valid and reliable instrument of rating the quality and risk of bias of a clinical trial. ${ }^{36,37}$ This scale consists of 11 items, with 10 criteria of internal validity and one external validity item, which is not counted into the total score.

Previous studies have interpreted the quality of trials according to the PEDro score, with excellent quality of 9 to 10 , good quality of 6 to 8 , fair quality of 4 to 5 , and poor quality of 4 or less. ${ }^{23}$ Additionally, another systematic review regarding exercise intervention on balance performance of older adults categorized trials with a score of 6 or above as high quality. ${ }^{4}$ Sherrington and colleagues have pointed out the difficulty of performing a double-blind procedure in interventions may limit the maximum PEDro score that a project can receive. ${ }^{38}$ Considering the interpretation of the quality score adopted by relevant research as well as characteristics of exercise intervention trials, we use the score of 6 as a cutoff point for high-quality studies with low risk of bias. ${ }^{38-40} \mathrm{~A}$ trial scoring 4 to 6 suggests moderate quality while a study with a score of 3 or below is considered low quality with high risk of bias. Two authors (P.G. and S.A.) independently conducted the quality assessment. Any disagreement was resolved through discussion with other authors.

\section{Data extraction and synthesis}

Data extraction summarizes essential details of the reviewed studies, including age of participants, sample size, attrition rate, inclusion criteria, outcome measures, and intervention protocol. Sample size and attrition rate are two variables that provide information about adherence to the exergaming protocol. This can partly reflect participants' enjoyment during the game play. Inclusion criteria summarizes the requirements to be eligible to participate in a study. This item indicates an overall health status of the enrolled participants. Outcome measures demonstrate balance tests used in the studies. Each individual test may measure on a specific aspect of balance ability but combining those tests can establish a comprehensive view on exergaming-related effects on balance performance.

Intervention protocol summarizes details of the training modalities such as the platform used in a trial (i.e., Wii or Xbox), duration of a single session, frequency (i.e., number of sessions per week), and duration of the whole intervention. The protocol information is useful to identify dose/response relationships and, from a practical perspective, may provide valuable advice for health professionals who attempt to develop an exergame-based intervention program. Table 1 summarizes the extracted information of the included studies.

Balance control is highly task specific, suggesting that balance performance should be assessed in separate categories. ${ }^{4}$ The model proposed by Shumway-Cook and Woollacott divides balance abilities into four components, including static, dynamic, proactive, and reactive balance, which are primary outcome measures in our meta-analysis. ${ }^{41}$

Static balance can be represented by tasks that require single-leg standing or bilateral stance in a stationary posture. Force plate analysis measuring postural sway in anterior/ posterior $(\mathrm{A} / \mathrm{P})$ or medial/lateral $(\mathrm{M} / \mathrm{L})$ direction is considered a proxy for static balance. Timed-Up-and-Go (TUG) is a widely used clinical test of gait, balance, and functional mobility. To complete the test in a minimal amount of time, subjects need to improve stride length, increase gait frequency, and maintain balance during walking and turning. ${ }^{42}$
Thus, TUG is the main representative of dynamic balance. Proactive balance involves postural adjustment in a selfinitiated movement, with the Functional Reach test being selected as the preferred proxy. ${ }^{43,44}$ Reactive balance is associated with balance control in response to an unexpected perturbation. ${ }^{45}$ However, the current meta-analysis could not include reactive balance because of limited data and various output measures across the existing research.

Secondary outcome measures mainly focus on balance test battery and perceived balance control. Balance test batteries such as Berg Balance Scale (BBS) and Tinetti Balance Assessment provide quantitative analyses of an individual's balance ability. Perceived balance is often measured by selfreported surveys such as Activities-Specific Balance Confidence $(A B C)$ scale and Falls Efficacy Scale (FES), which assess an older adult's confidence level when performing daily activities. Previous research has reported a strong relationship between perceived balance abilities and actual performance. ${ }^{46}$

Besides those objective measures of balance capabilities, it is helpful to take balance-related self-efficacy into account. ${ }^{47}$ In addition to balance test batteries and perceived balance, Chair Stand Test is also included as a secondary measure of balance performance. The test is more than a measure of lower limb strength and endurance as recent studies have identified correlation to gait speed, sensorimotor processing, balance, and postural control. ${ }^{48,49}$ Therefore, we include Chair Stand Test as a supplementary measure, which may provide additional information about older adults' balance performance.

\section{Statistical analysis}

Statistical analysis was conducted in Comprehensive MetaAnalysis 2.2. As a measure of effect size to explore differences between exergaming groups and control groups, Hedges' $g$ was calculated based on quantitative data, including sample size, mean, and standard deviation of both groups at baseline test and posttest. For the studies which provided median, minimum, and maximum values, statistical methods were used to estimate mean and standard deviation. ${ }^{50,51}$ Pooled effect sizes were categorized as small (Hedges' $\mathrm{g}<0.2$ ), moderate $(0.2<$ Hedges' $\mathrm{g}<0.8)$, and large (Hedges' $\mathrm{g}>0.8) .^{52}$

Due to expected differences across the included studies (i.e., subjects, sample size, and intervention protocol), a random effects model was applied. ${ }^{53}$ Heterogeneity was considered likely if $I^{2}>25 \%$, with $25 \%<I^{2}<50 \%$ being regarded as moderate and $I^{2}>50 \%$ as considerable. ${ }^{29,54}$ Egger's regression test was performed to assess publication bias in the reviewed literature. Significance test was two-tailed, with $P$-value less than 0.05 as evidence for publication bias. ${ }^{53}$ All the above mentioned information would be summarized in Table 2.

\section{Results}

\section{Study characteristics}

Initial search across the six databases resulted in a total of 774 articles. The first phase of screening based on titles excluded 662 irrelevant items. A further examination on abstracts removed 90 articles for the following reasons: abstract only $(N=6)$, book chapter $(N=2)$, review $(N=12)$, not peer-reviewed article $(N=5)$, and no exergaming intervention $(N=65)$. There were 62 articles considered for full-text analysis. Forty-six articles were excluded because included texts were duplicate $(N=3)$; study 







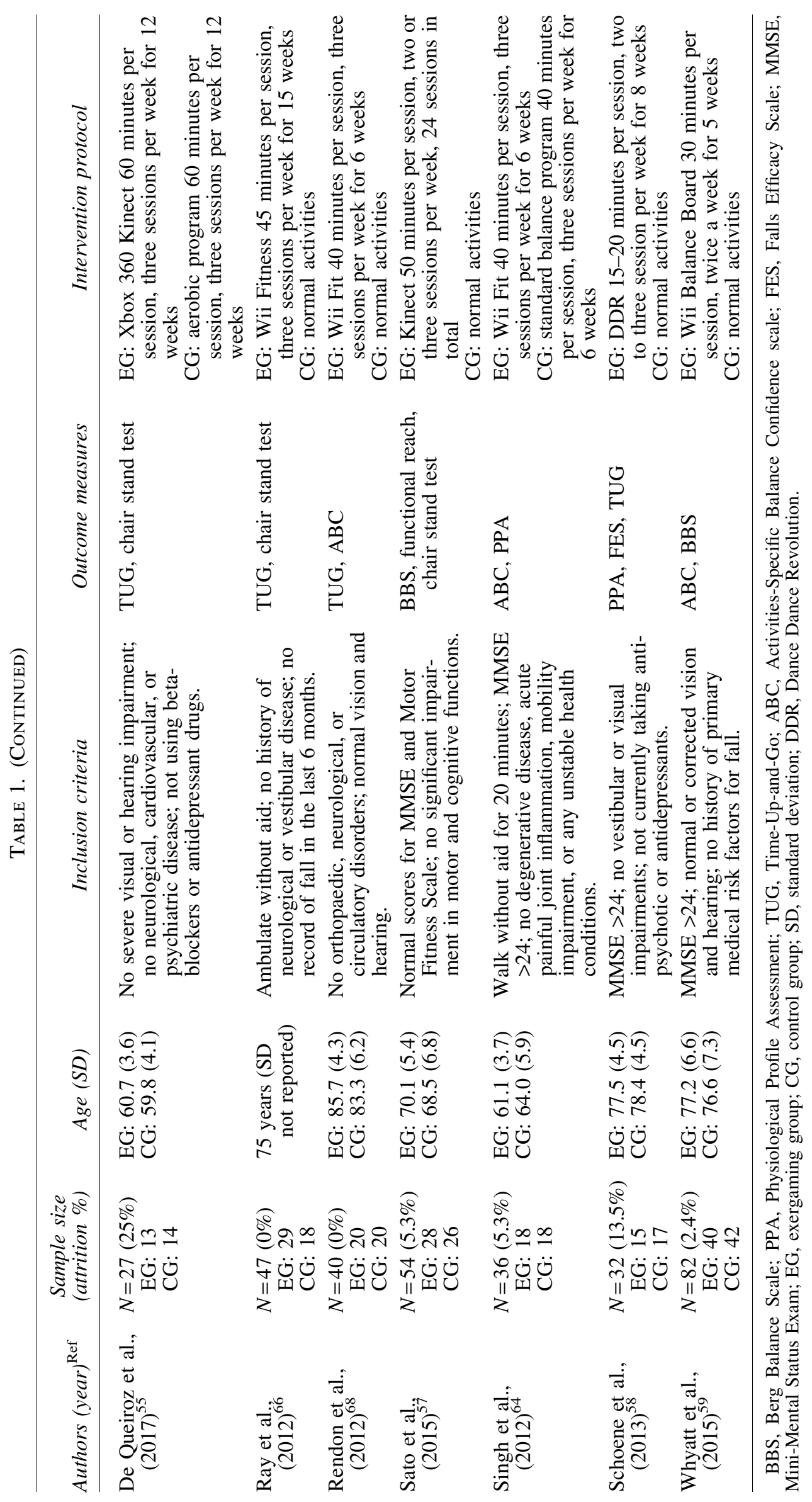


design was not experimental $(N=16)$, subjects were not healthy older adults $(N=15)$, outcome measures did not include balance performance $(N=9)$, and subjects were not randomly allocated in the trials $(N=3)$. The screening process finally retained 16 articles for qualitative and quantitative review. Figure 1 shows each step in the selection process.

The reviewed literature included a total of 646 subjects, with 322 subjects participating in the exergaming interventions and 324 subjects assigned to the control group. Subjects range in age between 60 and 86 years. Most studies kept a high retention. Only two studies reported an attrition rate above $15 \% .^{55,56}$

Further analysis was conducted to explore the reasons for dropping out of the exergaming intervention. The included studies reported 22 dropout cases in which 12 cases were because of participants' health issues (i.e., medication or illness), which made them either ineligible to the study or unable to attend the intervention program. ${ }^{5,57-61}$ Other dropout reasons include schedule conflicts $(N=4)$, travel distance $(N=3)$, and loss of interest $(N=3) .^{21,28,56,58}$ Given that participants of the included studies are above age of 60 , health issue could be a major dropout reason during the intervention. $^{62}$ Also, losing some participants because of schedule conflict or travel distance is hard to avoid in practice. In general, the included studies maintain a reasonable adherence rate in the exergaming group.

All included studies specified eligibility criteria for subjects participating in the intervention protocol. To ensure participants with adequate health fitness to perform an exergaming session, researchers conducted a comprehensive screening assessment on an individual's cognitive, motor, and sensory function.

The Mini-Mental Status Exam is the common instrument for mental health and cognitive assessment, with a score of 24 as the cutoff point for eligibility with respect to the cognitive function. ${ }^{28,57-59,61,63,64}$ Although older adults may experience considerable deficits in motor function, participants satisfying the eligible criteria must be able to stand and walk without aid. ${ }^{28,58,63-66}$ Sensory function is another major criterion, which requires no or minimal impairment in vision,
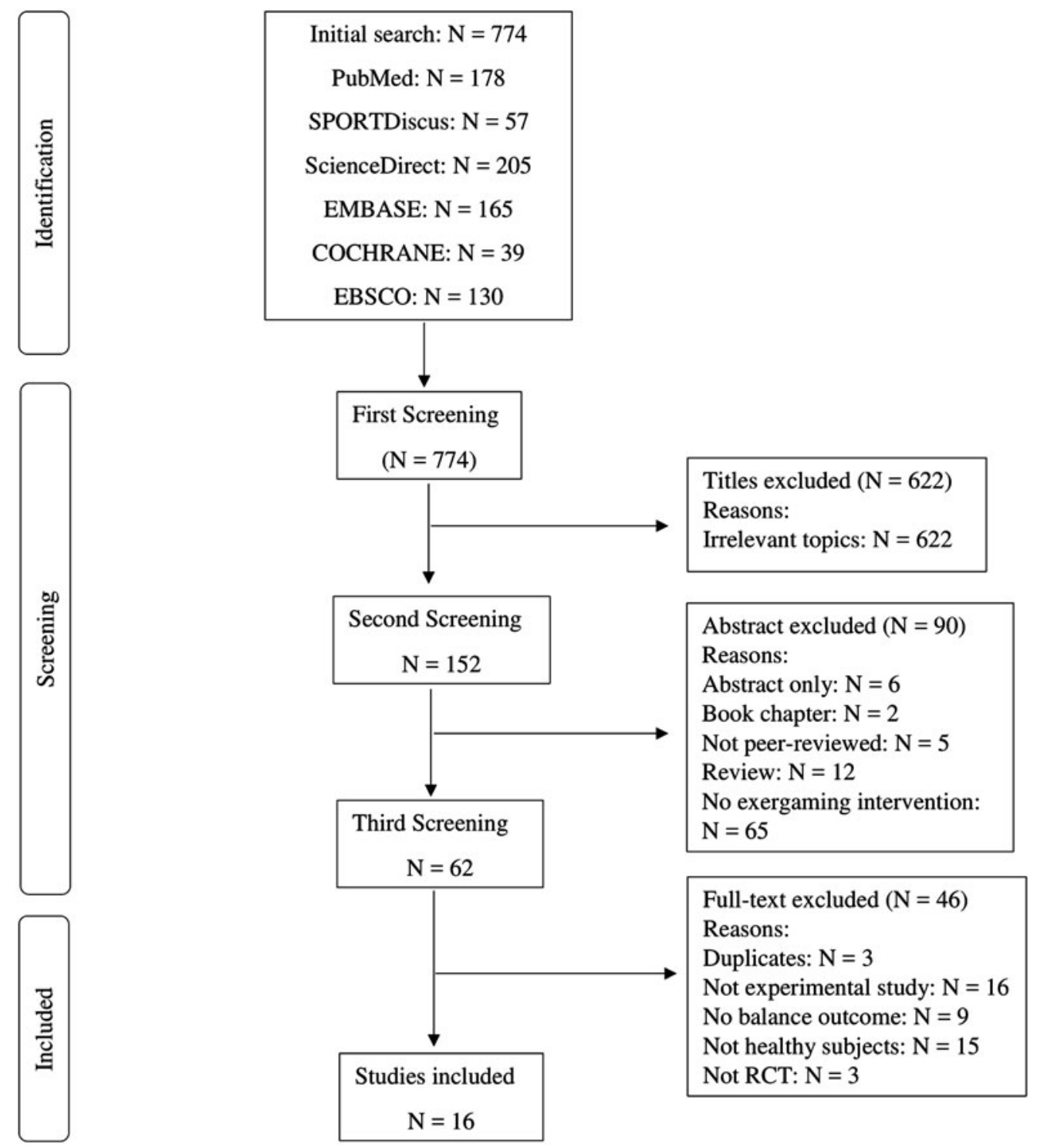

FIG. 1. Flowchart of the inclusion process. 
Table 2. Meta-analysis Examining Effects of Exergaming on Balance

\begin{tabular}{|c|c|c|c|c|c|c|c|}
\hline \multirow[b]{2}{*}{ Outcomes } & \multirow[b]{2}{*}{ Studies } & \multicolumn{2}{|c|}{ Sample size } & \multicolumn{2}{|c|}{ Meta-analysis } & \multicolumn{2}{|c|}{ Heterogeneity } \\
\hline & & Exergaming & Control & Hedges' $g$ & $95 \% C I$ & $\mathrm{I}^{2}$ & P-value \\
\hline Balance battery & 9 & 187 & 183 & 0.72 & $0.42-1.02$ & $25.27 \%$ & 0.07 \\
\hline Chair stand test & 6 & 127 & 131 & 0.78 & $0.26-1.30$ & $45.25 \%$ & 0.01 \\
\hline Dynamic balance & 10 & 194 & 195 & 0.36 & $0.17-0.56$ & $0 \%$ & 0.56 \\
\hline Perceived balance & 6 & 100 & 106 & 0.31 & $0.04-0.58$ & $0 \%$ & 0.85 \\
\hline Proactive balance & 3 & 40 & 39 & 0.54 & -0.12 to 1.20 & $44.68 \%$ & 0.16 \\
\hline Static balance & 6 & 65 & 68 & 0.20 & -0.09 to 0.48 & $0 \%$ & 0.75 \\
\hline
\end{tabular}

hearing, and vestibular system. ${ }^{21,55,56,58,59,63,65,67,68}$ Individuals with neuromuscular, neurological, cardiopulmonary, psychiatric disorders, or any unstable health issues were excluded from the intervention. Additionally, two of the reviewed trials considered people with fall experience in the past 6 months or 1 year ineligible for the inclusion criteria. ${ }^{66,67}$

Outcome measures take into account six aspects of balance performance, including static balance, dynamic balance, proactive balance, perceived balance, balance test batteries, and Chair Stand Test. Static balance was assessed by force plate $^{21,56,67}$ or Wii balance board, ${ }^{61}$ which recorded postural sway in $\mathrm{A} / \mathrm{P}$ and $\mathrm{M} / \mathrm{L}$ direction. TUG was the only approach to measuring dynamic balance. Of the 10 studies applied TUG test, three adopted 8-foot instead of 6-m test. ${ }^{30,55,68}$ The other procedures of the TUG test remained the same. Proactive balance was assessed only by functional reaching test. Selfreported questionnaires, $\mathrm{ABC}$ and FES, were utilized to reflect an individual's perceived balance. There were three types of balance test batteries to measure overall balance performance, including BBS $(N=6),{ }^{28,57,59,63,65,69}$ Tinetti test $(N=2),{ }^{56,69}$ and Physiological Profile Assessment (PPA), which consists of five functional tests involved in postural stability $(N=2) .{ }^{58,64}$ PPA predicts fall risk, with mild risk of fall in terms of a score between 0 and 1, moderate risk for a score between 1 and 2 , and considerable risk for scores above $2 .^{70}$

Information as to the intervention protocols suggests that the duration of a single session varied from 15 to 60 minutes with a mean of 40.6 minutes per session $($ Mean $=40.6$ minutes per session). A majority of trials consist of 30- to 45-minute exergaming-related activities per session. ${ }^{21,28,31,59,65-68,71}$ Subjects received 30 to 180 minutes of game play per week (Mean $=105.6$ minutes per week). Training frequency ranges between two and three sessions per week for a total of 3-15 weeks (Mean $=7.8$ weeks). In general, the included trials on average applied an exergaming program of 40-minute per session, two to three sessions per week for approximately 8 weeks.

\section{Quality assessment and risk of bias}

The average score of the 16 included trials is 6.4 out of 10 on the PEDro scale, suggesting moderate quality in study design (Table 3). Based on the predetermined level of quality assessment, six studies are considered high quality; 10 studies fall into the category of moderate quality. The included studies present a reasonable control over randomization, baseline similarity, attrition rate, intentional treatment given to both exergaming and control group, and reporting bias. However, a major limitation in the study design is the lack of blinding procedures. Only two trials used blinded therapists during intervention and evaluation, ${ }^{21,68}$ and two studies blinded assessors from group allocation. ${ }^{58,64}$ Notably, this limitation raises risk of bias in participant selection and performance evaluation.

\section{Effectiveness of exergaming on balance tests}

Effects of exergaming on static balance were assessed in four of the included studies, with 65 participants receiving

Table 3. Methodological Quality Assessment for Eligible Studies

\begin{tabular}{|c|c|c|c|c|c|c|c|c|c|c|c|}
\hline Authors, $(\text { Year })^{\operatorname{Ref}}$ & $R A$ & $C A$ & $S A B$ & $S B$ & $T B$ & $A B$ & $D R$ & ITA & $B C$ & $P M$ & Total \\
\hline ieryla and Dold, $(2013)^{65}$ & Yes & No & Yes & No & No & No & Yes & No & Yes & Yes & $5 / 10$ \\
\hline Franco et al., $(2012)^{69}$ & Yes & No & Yes & No & No & No & Yes & Yes & Yes & Yes & $6 / 1$ \\
\hline et al., $(2013)^{21}$ & Yes & Yes & Yes & Yes & Yes & No & Yes & Yes & Yes & Yes & $9 / 1$ \\
\hline Karahan et al., (2015) ${ }^{63}$ & Yes & No & Yes & No & No & No & Yes & Yes & Yes & Yes & 6 \\
\hline al., $(2012)^{30}$ & Yes & No & Yes & No & No & No & Yes & Yes & Yes & Yes & 6/1 \\
\hline Ordnung et al., $(2017)^{61}$ & Yes & No & Yes & Yes & No & No & Yes & Yes & Yes & Yes & $7 / 1$ \\
\hline$(2017)^{28}$ & Yes & Yes & No & No & No & No & Yes & No & Yes & Yes & $5 /$ \\
\hline$(2015)^{67}$ & Yes & No & Yes & No & No & No & Yes & Yes & Yes & Yes & $5 / 1$ \\
\hline tal., $(2012)^{56}$ & Yes & Yes & Yes & No & No & No & No & No & Yes & Yes & \\
\hline De Queiroz et al., $(2017)^{55}$ & Yes & Yes & Yes & Yes & No & No & No & No & Yes & Yes & $6 /$ \\
\hline$(2012)^{66}$ & Yes & No & Yes & No & No & No & Yes & Yes & Yes & Yes & $6 / 1$ \\
\hline Rendon et al., $(2012)^{68}$ & Yes & No & Yes & No & Yes & No & Yes & Yes & Yes & Yes & $7 / 1$ \\
\hline,$(2015)^{57}$ & Yes & Yes & Yes & No & No & No & Yes & Yes & Yes & Yes & $7 /$ \\
\hline Schoene et al., $(2013)^{58}$ & Yes & Yes & Yes & No & No & Yes & Yes & Yes & Yes & Yes & $8 / 1$ \\
\hline (2012 & Yes & No & Yes & Yes & No & Yes & Yes & Yes & Yes & Yes & 0 \\
\hline Whyatt et al., $(2015)^{59}$ & Yes & No & Yes & No & No & No & Yes & Yes & Yes & Yes & $6 / 1$ \\
\hline
\end{tabular}

RA, random allocation; $\mathrm{CA}$, concealed allocation; $\mathrm{SAB}$, similar at baseline; SB, subject blinded; TB, therapist blinded; AB, assessor blinded; DR, dropout rate; ITA, intention-to-treat analysis; $\mathrm{BC}$, between-group comparison; PM, points measures. 


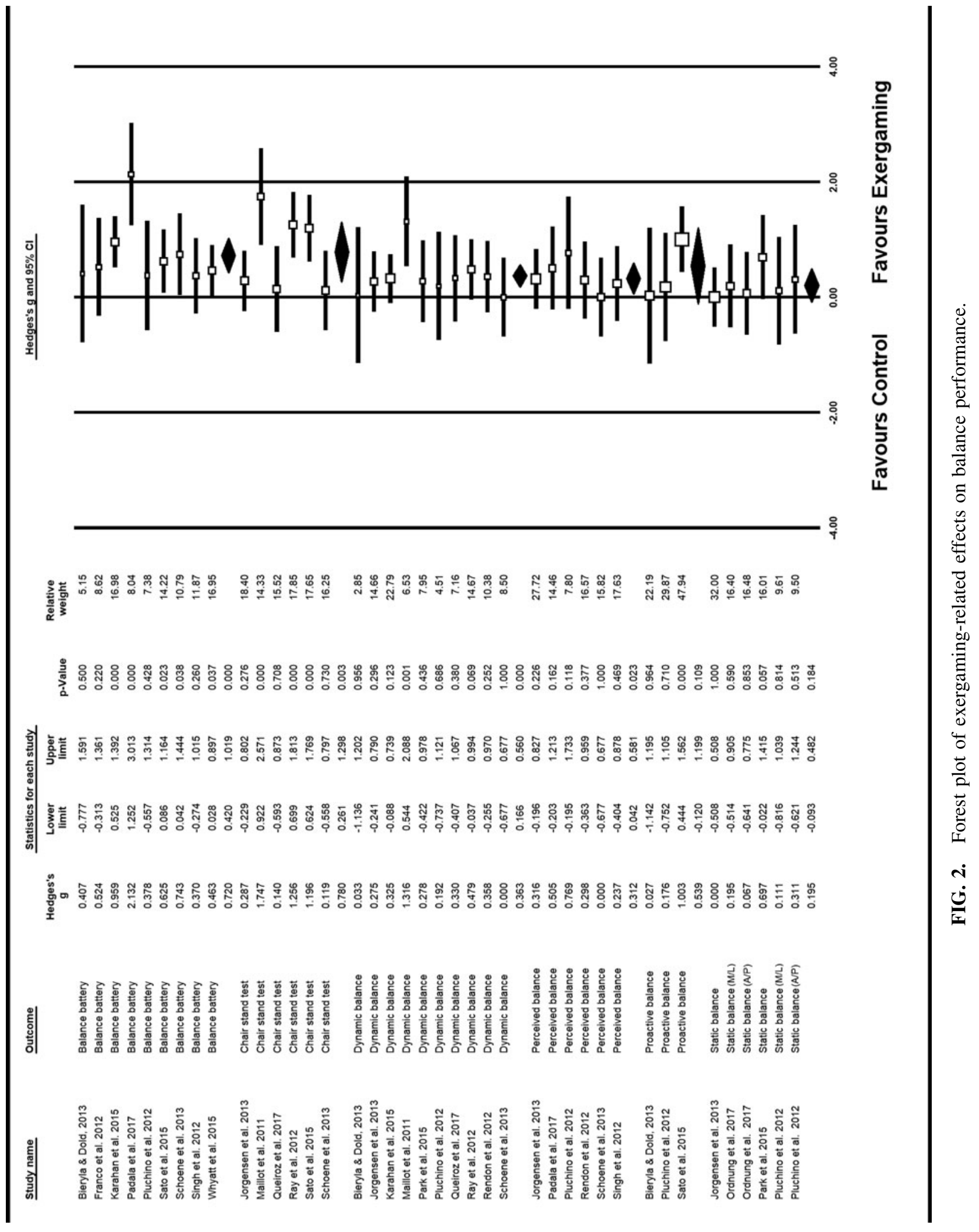


the exergaming intervention compared with 68 participants assigned to the control group (Table 2). ${ }^{21,56,61,67}$ Favorable outcomes were observed in the exergaming group, but the improvement in static balance performance did not reach the significant level (Hedges' $\mathrm{g}=0.20,95 \% \mathrm{CI}=-0.09$ to 0.48 , $P=0.18, I^{2}=0$; Fig. 2). No publication bias was identified in the studies as Egger's regression test was nonsignificant $(d f=4, t=0.87, P=0.44)$.

Ten of the reviewed studies involving a total of 389 subjects (exergaming $=194$, control $=195$; Table 2$)$ measured time to complete the TUG, which is a proxy for dynamic balance test. $^{21,30,55,56,58,63,65-68}$ Exergaming intervention indicated a moderate effect on TUG performance (Hedges' $\mathrm{g}=0.36,95 \%$ $\mathrm{CI}=0.26-1.30, P<0.001, I^{2}=0$; Fig. 2), suggesting that exergames are effective in improving dynamic balance for older adults. Egger's regression test found no evidence on publication bias in the studies $(d f=8, t=0.07, P=0.94)$.

Effects of exergaming intervention on proactive balance were examined in three studies, which included 79 subjects in total, with 40 in the exergaming group and 39 in the control group (Table 2). ${ }^{56,57,65}$ Exergaming shows a moderate effect size for older adults in proactive balance performance, but the improvement observed in the exergaming group is nonsignificant (Hedges' $\mathrm{g}=0.54,95 \% \mathrm{CI}=-0.12$ to $1.20, P=0.11, I^{2}=44.68 \%$; Fig. 2). Further analysis on $\mathrm{Eg}-$ ger's regression test suggests no publication bias in the relevant $(d f=1, t=4.80, P=0.13)$.

Balance test batteries involved nine studies composed of 187 participants in the exergaming group versus 183 participants in the control group (Table 2). ${ }^{28,56-59,63-65,69} \mathrm{Eg}$ ger's regression test of publication bias was not significant $(d f=7, t=0.27, P=0.80)$. Pooled effect size is significant (Hedges' $\mathrm{g}=0.72, \quad 95 \% \quad \mathrm{CI}=0.42-1.02, \quad P<0.001$, $I^{2}=25.27 \%$; Fig. 2). The result suggests that subjects receiving exergaming intervention indicate a significant improvement in the overall balance assessment.

Six of the studies examined self-report confidence in fall prevention by means of the FES and ABC scale. $^{21,28,56,58,64,68}$ The included studies contained 100 subjects in the exergaming group and 106 subjects in the control group (Table 2). Meta-analysis indicated a moderate effect with no heterogeneity affecting the results (Hedges' $\mathrm{g}=0.31,95 \% \mathrm{CI}=0.04-0.58, P=0.02, I^{2}=0$; Fig. 2). No publication bias was observed in Egger's regression test $(d f=4, t=1.06, P=0.35)$. It suggests that exergaming interventions are helpful to establish more confidence in balance control, and less fear of falling for older adults when performing daily activities.

Subjects participating in exergaming intervention $(N=127)$ had significantly better performance than their counterparts in the control group $(N=131)$ in Chair Stand Test (Table 2). Hedges' g suggested a moderate to high effect size. However, it is worth noticing that $I^{2}$ showed a considerable heterogeneity across the studies (Hedges' $\mathrm{g}=0.78,95 \% \mathrm{CI}=0.26-$ 1.30, $P=0.003, I^{2}=45.24 \%$; Fig. 2). ${ }^{21,30,55,57,58,66}$ Egger's test was not significant, suggesting no publication bias in the studies $(d f=4, t=0.24, P=0.82)$.

\section{Discussion}

Exercise is effective in improving balance control and thus reducing risk of fall in older adults. ${ }^{12,13,72} \mathrm{~A}$ systematic re- view on 54 RCTs indicates significant effects of exercise on fall prevention. The included trials were undertaken in a variety of forms, including strength training, endurance training, flexibility and agility training, and walking. ${ }^{73}$ However, low adherence has been a major issue in clinical practice, which prevents older adults from achieving longterm benefits. ${ }^{74,75}$ As a promising instrument for health promotion, exergames provide interactive game settings to potentially motivate continuous engagement. ${ }^{21}$ Feedback received from participants in the previous studies suggests that using the exergames is more appealing and rewarding than traditional exercises. ${ }^{76}$

The current meta-analysis on 16 RCTs identified favorable outcomes in the exergaming group compared with the control group. Specifically, significant effect was found in both functional performance test and balance confidence assessment with respect to dynamic balance, perceived balance, Chair Stand Test, and balance test batteries. However, the effect in the static balance as well as proactive balance was nonsignificant. A reasonable explanation for the nonsignificant effect on static balance may be attributed to potential ceiling effects in the test. ${ }^{4}$ Healthy older adults tend to have a high score at the baseline test, leaving limited room for further improvement in a posttest. Such an explanation might be supported by the fact that significant effects were identified in the tests requiring advanced balance control. For example, the Chair Stand Test requires participants to repeatedly perform the sit-and-stand movement, indicating the level of functional strength and endurance in the lower extremity muscles in combination with balance control. ${ }^{21,77}$

Additionally, the TUG is a test of functional ability involving gait speed, stride length, and balance in locomotion. ${ }^{78,79}$ Both tests require more than keeping balance in a static stance. Therefore, significant effects associated with exergaming interventions are more likely to be observed in these tests than changes in static stance. Exergaming-related effects on proactive balance proxied by functional reaching test indicate a nonsignificant, but moderate effect size (Hedges' $g=0.54$ ). The nonsignificant result may be explained by the small sample size as only three trials were available for data analysis. Given the limited number of studies with respect to the proactive balance, a moderate effect size should be interpreted as meaningful improvement particularly for older adults.

All included studies for literature review were based on RCTs, which provided stronger evidence than other types of study design. To determine feasibility and value of a relatively new intervention, stringent research methods should be adopted. ${ }^{74,80}$ Previous research usually excluded study designs with scores lower than $4 .^{72}$ In the current systematic review, all included studies had a score of 5 or above. Quality assessment identified a moderate to high quality in study design (6.4 out 10 points), suggesting a relatively low risk of bias in the reviewed articles. Therefore, meta-analysis was conducted on all the included studies. However, trials with a score of 5 still had limitations in the study design, especially in concealment and blinding procedures, which could reduce credibility of meta-analysis results. For this reason, further evidence based on high-quality trials is still needed in future.

The included protocols on average consist of two to three 40-minute sessions per week for approximately 8 weeks. This finding may provide recommendations with respect to 
duration of a single session, frequency, and period. However, considerable variations in training period (3-15 weeks) and duration of a single session (15-60 minutes) make it impractical to determine the training volume for optimal treatment effects. Comparable information may be found in another meta-analysis examining the dose/response relationship on balance training in healthy older adults. Researchers observed the largest improvement in overall balance performance when training modalities were conducted three times per week for a total of 11 or 12 weeks, with 31-45 minutes of exercise in a single session. ${ }^{4}$

Consistency between the two studies can be found in the frequency and duration of a single session, whereas discrepancy exists in the training period. It suggests that the training periods of the exergaming interventions ( 8 weeks) are relatively short in comparison with the balance training modalities (12 weeks). Whether greater benefits can be achieved through a longer period of exergaming intervention remains unclear. Further investigation is required to identify the relationship between training volume of exergaming intervention and corresponding effects.

Three of the reviewed studies compared exergaming groups with active control groups receiving exercise programs, including standardized balance training, aerobic exercise, and ball practice program. ${ }^{55,56,67}$ Results of the three studies indicated that exergaming-induced effects on balance control were comparable with the benefits in a standard exercise program. A possible reason may be attributed to the similar features shared between exergames and exercise. Research has found that exergaming increases heart rate, oxygen consumption, and energy expenditure at a magnitude comparable to light- to moderate-intensity physical activity. ${ }^{24}$ Given the exercise-related effects on balance control in healthy older adults, similar benefits should be observed with exergaming practice. ${ }^{4,81,82}$

On the other hand, sports exergames, such as soccer and ski, require adequate motor control for rapid and accurate movements. ${ }^{55}$ Also, games using the Wii Balance Board provide a feasible form of practice of balance control when participants make attempts during the game to lean toward different directions and shift their center of mass to the limits of their base of support. ${ }^{32}$

To improve game performance, participants are motivated to continually adjust their movements according to the visual stimuli presented on the screen. Repetitive muscle actions and joint motions enhance proprioception and spatial perception, which contribute to improved balance performance in older adults. The current meta-analysis provides supportive information as to utilizing exergaming as a supplementary resource to conventional exercise intervention. ${ }^{32,83}$ Based on the promising findings of the included studies, it is recommended to encourage application of exergames to clinical practice. By integrating exergaming sessions into standard balance exercise, health professionals may optimize the effectiveness of an intervention.

\section{Limitation and future direction}

Data coding and synthesis was based on the theoretical framework proposed by Shumway-Cook and Woollacott, ${ }^{41}$ which provided a comprehensive view on different aspects of balance abilities, including static, dynamic, proactive, and reactive balance. However, reactive balance was not accounted for in the current review due to the limited number of studies as well as discrepancies with respect to outcome measures, study design, and type of intervention.

Reactive balance is important when people try to restore balance in an unpredicted perturbation. ${ }^{84}$ Lacking measures of reactive balance is a limitation of the current review, as falls often result from an inadequate reactive response to an unexpected disturbance. ${ }^{85}$ According to the existing research, reactive balance is measured by capturing Center of Pressure (CoP) displacement after a sudden perturbation. Participants stand on a platform which can slide forward or backward to cause a sudden postural change. ${ }^{86,87}$ Researchapplied exercise training identified decreased $\mathrm{CoP}$ displacement, which suggested improved postural control in response to the perturbations.

Another commonly used assessment of reactive balance is based on gait perturbation. A participant walks on a hard surface walkway during which a soft surface (i.e., foam) is placed without the participant's knowledge. Parameters such as CoP displacement and joint moment reflect stability control in response to the unexpected perturbation. ${ }^{88}$ Given the fact that assessments utilized in the included studies are mostly concerned with balance control under self-initiated conditions, it would be meaningful if future research could provide an insight into exergaming-associated effects on reactive balance in older adults.

Another limitation might be attributed to the fact that outcome measures of the reviewed studies are mostly based on clinical tests. Valid and reliable clinical tests have been widely used because they are cost effective, time efficient, and easy to administer. In addition to the commonly used clinical balance tests, biomechanical assessments should receive more attention in future studies. A sophisticated biomechanical instrument provides objective assessments of balance control under various conditions. As discussed earlier, a sliding force plate can be used to simulate an unexpected perturbation while the walkway is suitable to assess dynamic and reactive balance in locomotion.

However, the only biomechanical measure utilized in the reviewed studies is the $\mathrm{CoP}$ displacement in a static stance. Maillot et al. ${ }^{89}$ investigated another biomechanical measure of balance performance, which can be applied to future research. In the study investigating exergaming-associated influence on the gait pattern of older adults, Maillot et al. ${ }^{86}$ used the force plate to record the braking force in walking. The amount of force in the heel strike is an important indicator of balance control in locomotion. However, aging has an impact on reducing the braking force. Increased braking force after the exergaming intervention suggests a positive outcome of counteracting age-related decline in balance control. ${ }^{89}$

\section{Conclusion}

The current systematic review and meta-analysis identified exergaming-associated improvements in balance function and confidence among healthy older adults. Statistical tests of publication bias in all comparisons were not significant. It is worth pointing out that heterogeneity was identified in balance test battery, Chair Stand Test, and proactive balance, suggesting that the results should be interpreted with caution. Researchers should develop study designs that 
reflect reactive balance and utilize biomechanical measures in future studies. Despite limitations of the current review, this meta-analysis based on 16 experimental studies still provides evidence that supports using exergaming intervention as a supplementary instrument to traditional balance training for older adults.

\section{Acknowledgment}

The authors would like to express gratitude for the help and support from the Student Review Committee of the Games for Health Journal in conducting this review.

\section{Author Disclosure Statement}

The authors declared no potential conflicts of interest and received no financial support with respect to the research or publication of this article.

\section{References}

1. Kanekar N, Aruin AS. Aging and balance control in response to external perturbations: Role of anticipatory and compensatory postural mechanisms. Age 2014; 36:9621.

2. Sturnieks DL, St George R, Lord SR. Balance disorders in the elderly. Clin Neurophysiol 2008; 38:467-478.

3. Fling BW, Dutta GG, Schlueter H, et al. Associations between proprioceptive neural pathway structural connectivity and balance in people with multiple sclerosis. Front Hum Neurosci 2014; 8:814.

4. Lesinski M, Hortobágyi T, Muehlbauer T, et al. Effects of balance training on balance performance in healthy older adults: A systematic review and meta-analysis. Sports Med 2015; 45:1721-1738.

5. Bergen G. Falls and fall injuries among adults aged $\geq 65$ years-United States, 2014. MMWR Morb Mortal Wkly Rep 2016; 65:993-998.

6. Burns E, Kakara R. Deaths from Falls Among Persons Aged $\geq 65$ Years-United States, 2007-2016. Morb Mortal Wkly Rep 2018; 67:509.

7. Vellas BJ, Wayne SJ, Romero LJ, et al. Fear of falling and restriction of mobility in elderly fallers. Age Ageing 1997; 26:189-193.

8. Decullier E, Couris C, Beauchet O, et al. Falls' and fallers' profiles. J Nutr Health Aging 2010; 14:602-608.

9. Salkeld G, Ameratunga SN, Cameron I, et al. Quality of life related to fear of falling and hip fracture in older women: A time trade off studyCommentary: Older people's perspectives on life after hip fractures. BMJ 2000; 320:341-346.

10. Li F, Fisher KJ, Harmer P, et al. Fear of falling in elderly persons: Association with falls, functional ability, and quality of life. J Gerontol B Psychol Sci Soc Sci 2003; 58: P283-P290.

11. Garber CE, Blissmer B, Deschenes MR, et al. Quantity and quality of exercise for developing and maintaining cardiorespiratory, musculoskeletal, and neuromotor fitness in apparently healthy adults: Guidance for prescribing exercise. Med Sci Sports Exerc 2011; 43:1334-1359.

12. Rogers ME, Rogers NL, Takeshima N, Islam MM. Methods to assess and improve the physical parameters associated with fall risk in older adults. Prev Med 2003; 36:255-264.

13. Carter ND, Kannus P, Khan K. Exercise in the prevention of falls in older people. Sports Med 2001; 31:427-438.
14. Melzer I, Benjuya N, Kaplanski J. Postural stability in the elderly: A comparison between fallers and non-fallers. Age Ageing 2004; 33:602-607.

15. Tinetti ME. Preventing falls in elderly persons. N Engl J Med 2003; 348:42-49.

16. Rugelj D. The effect of functional balance training in frail nursing home residents. Arch Gerontol Geriatr 2010; 50: 192-197.

17. Seidler RD, Martin PE. The effects of short term balance training on the postural control of older adults. Gait Posture 1997; 6:224-236.

18. Perkin OJ, Travers RL, Gonzalez JT, et al. Exercise strategies to protect against the impact of short-term reduced physical activity on muscle function and markers of health in older men: Study protocol for a randomised controlled trial. Trials 2016; 17:381.

19. Day L, Fildes B, Gordon I, et al. Randomised factorial trial of falls prevention among older people living in their own homes. BMJ 2002; 325:128.

20. Chao Y-Y, Scherer YK, Montgomery CA. Effects of using Nintendo Wii ${ }^{\mathrm{TM}}$ exergames in older adults: A review of the literature. J Aging Health 2015; 27:379-402.

21. Jorgensen MG, Laessoe U, Hendriksen C, et al. Efficacy of Nintendo Wii training on mechanical leg muscle function and postural balance in community-dwelling older adults: A randomized controlled trial. J Gerontol A Biomed Sci Med Sci 2013; 68:845-852.

22. Petersen T, Larsen K, Jacobsen S. One-year follow-up comparison of the effectiveness of McKenzie treatment and strengthening training for patients with chronic low back pain: Outcome and prognostic factors. Spine 2007; 32: 2948-2956.

23. Cheok G, Tan D, Low A, Hewitt J. Is Nintendo Wii an effective intervention for individuals with stroke? A systematic review and meta-analysis. J Am Med Dir Assoc 2015; 16:923-932.

24. Peng W, Lin J-H, Crouse J. Is playing exergames really exercising? A meta-analysis of energy expenditure in active video games. Cyberpsychol Behav Soc Netw 2011; 14: 681-688.

25. Graves LE, Ridgers ND, Williams K, et al. The physiological cost and enjoyment of Wii Fit in adolescents, young adults, and older adults. J Phys Act Health 2010; 7:393-401.

26. Hilton CL, Cumpata K, Klohr C, et al. Effects of exergaming on executive function and motor skills in children with autism spectrum disorder: A pilot study. Am J Occup Ther 2014; 68:57-65.

27. Haddock BL, Siegel SR, Wikin LD. The addition of a video game to stationary cycling: The impact on energy expenditure in overweight children. Open Sports Sci J 2009; 2:42.

28. Padala KP, Padala PR, Lensing SY, et al. Efficacy of WiiFit on static and dynamic balance in community dwelling older veterans: A randomized controlled pilot trial. J Aging Res 2017; 2017:4653635.

29. Tahmosybayat R, Baker K, Godfrey A, et al. A systematic review and meta-analysis of outcome measures to assess postural control in older adults who undertake exergaming. Maturitas 2017; 98:35-45.

30. Maillot P, Perrot A, Hartley A. Effects of interactive physical-activity video-game training on physical and cognitive function in older adults. Psychol Aging 2012; 27:589.

31. Nicholson VP, McKean M, Lowe J, et al. Six weeks of unsupervised Nintendo Wii Fit gaming is effective at im- 
proving balance in independent older adults. J Aging Phys Act $2015 ; 23: 153-158$.

32. Laufer Y, Dar G, Kodesh E. Does a Wii-based exercise program enhance balance control of independently functioning older adults? A systematic review. Clin Interv Aging 2014; 9:1803.

33. Group GW. Grading quality of evidence and strength of recommendations. BMJ 2004; 328:1490.

34. Djulbegovic B, Guyatt GH. Progress in evidence-based medicine: A quarter century on. Lancet 2017; 390:415423.

35. Higgins JP, Altman DG, Gøtzsche PC, et al. The Cochrane Collaboration's tool for assessing risk of bias in randomised trials. BMJ 2011; 343:d5928.

36. Sherrington C, Herbert R, Maher C, Moseley A. PEDro. A database of randomized trials and systematic reviews in physiotherapy. Man Ther 2000; 5:223-226.

37. Christopher GM, Catherine S, Robert DH, et al. Reliability of the PEDro scale for rating quality of randomized controlled trials. Phys Ther 2003; 83:713-721.

38. Sherrington C, Moseley AM, Herbert RD, et al. Ten years of evidence to guide physiotherapy interventions: Physiotherapy Evidence Database (PEDro). Br J Sports Med 2010; 44:836-837.

39. Armijo-Olivo S, Costa BRD, Cummings GG, et al. PEDro or Cochrane to Assess the Quality of Clinical Trials? A MetaEpidemiological Study. PLoS One 2015; 10:e0132634.

40. Fang Q, Aiken CA, Fang C, Pan Z. Effects of Exergaming on Physical and Cognitive Functions in Individuals with Autism Spectrum Disorder: A Systematic Review. Games Health J 2019; 8:74-84.

41. Shumway-Cook A, Woollacott MH. Motor Control: Translating Research into Clinical Practice. Philadelphia, United States: Lippincott Williams \& Wilkins; 2007.

42. Podsiadlo D, Richardson S. The timed "Up \& Go": A test of basic functional mobility for frail elderly persons. J Am Geriatr Soc 1991; 39:142-148.

43. Huxham FE, Goldie PA, Patla AE. Theoretical considerations in balance assessment. Aust J Physiother 2001; 47: 89-100.

44. Muehlbauer T, Besemer C, Wehrle A, et al. Relationship between strength, balance and mobility in children aged 7 10 years. Gait Posture 2013; 37:108-112.

45. Granacher U, Muehlbaue T, Zahner L, et al. Comparison of traditional and recent approaches in the promotion of balance and strength in older adults. Sports Med 2011; 41:377-400.

46. Myers AM, Powell LE, Maki BE, et al. Psychological indicators of balance confidence: Relationship to actual and perceived abilities. J Gerontol A Biol Sci Med Sci 1996; 51:M37-M43.

47. Liu-Ambrose T, Khan KM, Eng JJ, et al. Balance confidence improves with resistance or agility training. Gerontology 2004; 50:373-382.

48. Lord SR, Murray SM, Chapman K, et al. Sit-to-stand performance depends on sensation, speed, balance, and psychological status in addition to strength in older people. $\mathrm{J}$ Gerontol A Biol Sci Med Sci 2002; 57:M539-M543.

49. Whitney SL, Wrisley DM, Marchetti GF, et al. Clinical measurement of sit-to-stand performance in people with balance disorders: Validity of data for the Five-Times-Sitto-Stand Test. Phys Ther 2005; 85:1034-1045.

50. Luo D, Wan X, Liu J, Tong T. Optimally estimating the sample mean from the sample size, median, mid-range, and/or mid-quartile range. Stat Methods Med Res 2018; 27: 1785-1805.

51. Wan X, Wang W, Liu J, Tong T. Estimating the sample mean and standard deviation from the sample size, median, range and/or interquartile range. BMC Med Res Methodol $2014 ; 14: 135$

52. Stanmore E, Stubbs B, Vancampfort D, et al. The effect of active video games on cognitive functioning in clinical and non-clinical populations: A meta-analysis of randomized controlled trials. Neurosci Biobehav Rev 2017; 78:34-43.

53. DerSimonian R, Kacker R. Random-effects model for meta-analysis of clinical trials: An update. Contemp Clin Trials 2007; 28:105-114.

54. Higgins JP, Thompson SG, Deeks JJ, Altman DG. Measuring inconsistency in meta-analyses. BMJ 2003; 327:557.

55. De Queiroz BM, Borgatto AF, Barbosa AR, Guimarães AV. Exergame vs. Aerobic Exercise and Functional Fitness of Older Adults: A Randomized Controlled Trial. J Phys Educ Sport 2017; 17:740.

56. Pluchino A, Lee SY, Asfour S, et al. Pilot study comparing changes in postural control after training using a video game balance board program and 2 standard activity-based balance intervention programs. Arch Phys Med Rehabil 2012; 93:1138-1146.

57. Sato K, Kuroki K, Saiki S, Nagatomi R. Improving walking, muscle strength, and balance in the elderly with an exergame using Kinect: A randomized controlled trial. Games Health J 2015; 4:161-167.

58. Schoene D, Lord SR, Delbaere K, et al. A randomized controlled pilot study of home-based step training in older people using videogame technology. PLoS One 2013; 8: e57734.

59. Whyatt C, Merriman NA, Young WR, et al. A Wii bit of fun: A novel platform to deliver effective balance training to older adults. Games Health J 2015; 4:423-433.

60. Jorgensen M, Rathleff MS, Laessoe U, et al. Time-of-day influences postural balance in older adults. Gait Posture 2012; 35:653-657.

61. Ordnung M, Hoff M, Kaminski E, et al. No overt effects of a 6-week exergame training on sensorimotor and cognitive function in older adults. A preliminary investigation. Front Hum Neurosci 2017; 11:160.

62. Picorelli AMA, Pereira LSM, Pereira DS, et al. Adherence to exercise programs for older people is influenced by program characteristics and personal factors: A systematic review. J Physiother 2014; 60:151-156.

63. Karahan AY, Tok F, Taskin H, et al. Effects of exergames on balance, functional mobility, and quality of life of geriatrics versus home exercise programme: Randomized controlled study. Central Eur J Public Health 2015; 23:S14.

64. Singh DK, Rajaratnam BS, Palaniswamy V, et al. Participating in a virtual reality balance exercise program can reduce risk and fear of falls. Maturitas 2012; 73:239-243.

65. Bieryla KA, Dold NM. Feasibility of Wii Fit training to improve clinical measures of balance in older adults. Clin Interv Aging 2013; 8:775.

66. Ray C, Melton F, Ramirez R, Keller D. The effects of a 15week exercise intervention on fitness and postural control in older adults. Activities Adaptation Aging 2012; 36:227241.

67. Park E-C, Kim S-G, Lee C-W. The effects of virtual reality game exercise on balance and gait of the elderly. J Phys Ther Sci 2015; 27:1157-1159. 
68. Rendon AA, Lohman EB, Thorpe D, et al. The effect of virtual reality gaming on dynamic balance in older adults. Age Ageing 2012; 41:549-552.

69. Franco JR, Jacobs K, Inzerillo C, Kluzik J. The effect of the Nintendo Wii Fit and exercise in improving balance and quality of life in community dwelling elders. Technol Health Care 2012; 20:95-115.

70. Lord SR, Menz HB, Tiedemann A. A physiological profile approach to falls risk assessment and prevention. Phys Ther 2003; 83:237-252.

71. Merriman NA, Whyatt C, Setti A, et al. Successful balance training is associated with improved multisensory function in fall-prone older adults. Comput Hum Behav 2015; 45: 192-203.

72. Chou C-H, Hwang C-L, Wu Y-T. Effect of exercise on physical function, daily living activities, and quality of life in the frail older adults: a meta-analysis. Arch Phys Med Rehabil 2012; 93:237-244.

73. Sherrington C, Tiedemann A, Fairhall N, et al. Exercise to prevent falls in older adults: an updated meta-analysis and best practice recommendations. N S W Public Health Bull 2011; 22:78-83.

74. Van Diest M, Lamoth CJ, Stegenga J, et al. Exergaming for balance training of elderly: state of the art and future developments. J Neuroeng Rehabil 2013; 10:101.

75. Sherrington C, Whitney JC, Lord SR, et al. Effective exercise for the prevention of falls: a systematic review and meta-analysis. J Am Geriatr Soc 2008; 56:2234-2243.

76. Fitzgerald D, Trakarnratanakul N, Smyth B, Caulfield B. Effects of a wobble board-based therapeutic exergaming system for balance training on dynamic postural stability and intrinsic motivation levels. J Orthop Sports Phys Ther 2010; 40:11-19.

77. Jones CJ, Rikli RE, Beam WC. A 30-s chair-stand test as a measure of lower body strength in community-residing older adults. Res Q Exerc Sport 1999; 70:113-119.

78. Beauchet O, Fantino B, Allali G, et al. Timed Up and Go test and risk of falls in older adults: A systematic review. $\mathrm{J}$ Nutr Health Aging 2011; 15:933-938.

79. Bohannon RW. Reference values for the timed up and go test: a descriptive meta-analysis. J Geriatr Phys Ther 2006; 29:64-68.

80. Larsen LH, Schou L, Lund HH, Langberg H. The physical effect of exergames in healthy elderly-a systematic review. Games Health J 2013; 2:205-212.
81. Weerdesteyn V, Rijken H, Geurts AC, et al. A five-week exercise program can reduce falls and improve obstacle avoidance in the elderly. Gerontology 2006; 52:131-141.

82. Sherrington C, Michaleff ZA, Fairhall N, et al. Exercise to prevent falls in older adults: an updated systematic review and meta-analysis. Br J Sports Med 2017; 51:1750-1758.

83. Gao Z, Chen S. Are field-based exergames useful in preventing childhood obesity? A systematic review. Obes Rev 2014; 15:676-691.

84. Carty CP, Cronin NJ, Nicholson D, et al. Reactive stepping behaviour in response to forward loss of balance predicts future falls in community-dwelling older adults. Age Ageing 2014; 44:109-115.

85. Tseng S-C, Stanhope SJ, Morton SM. Impaired reactive stepping adjustments in older adults. J Gerontol A Biomed Sci Med Sci 2009; 64:807-815.

86. Rossi LP, Pereira R, Brandalize M, Gomes ARS. The effects of a perturbation-based balance training on the reactive neuromuscular control in community-dwelling older women: A randomized controlled trial. Hum Movement 2013; 14:238-246.

87. De Freitas PB, Knight CA, Barela JA. Postural reactions following forward platform perturbation in young, middleage, and old adults. J Electromyogr Kinesiol 2010; 20:693700 .

88. Bierbaum S, Peper A, Arampatzis A. Exercise of mechanisms of dynamic stability improves the stability state after an unexpected gait perturbation in elderly. Age 2013; 35: 1905-1915.

89. Maillot P, Perrot A, Hartley A, Do M-C. The braking force in walking: Age-related differences and improvement in older adults with exergame training. J Aging Phys Act 2014; 22:518-526.
Address correspondence to: Chao Fang, PhD Department of Pharmacology the Fourth Military Medical University 169 Changle West Road $X i$ 'an 710032 China

E-mail: fang1989@fmmu.edu.cn 